\title{
A novel OFET-based biosensor for the selective and sensitive detection of lactate levels
}

Tsuyoshi Minami,* Tsubasa Sato, Tsukuru Minamiki, Kenjiro Fukuda, Daisuke Kumaki and Shizuo Tokito

Research Center for Organic Electronics (ROEL)

Graduate School of Science and Engineering, Yamagata University

4-3-16 Jonan, Yonezawa, Yamagata 992-8510 Japan

Tel: +81-238-26-3594; Fax: +81-238-26-3788

E-mail: tminami@yz.yamagata-u.ac.jp

\begin{abstract}
Biosensors based on organic field effect transistors (OFETs) are one of the more promising device applications in organic electronics. However, OFET-based biosensors are still in their early stages of development compared to other electrochemical biosensors. This study is the first to report on an extended-gate type organic field effect transistor (OFET) for lactate detection in aqueous media. Here, the extended-gate electrode of the OFET was modified with layers of a lactate oxidase and a horseradish peroxidase osmium-redox polymer on a flexible plastic film substrate for an enzymatic redox reaction of lactate. The device exhibited both high selectivity and sensitivity. The limit of detection (LOD) and the limit of quantification (LOQ) were estimated to be 66 $\mathrm{nM}$ and $220 \mathrm{nM}$, respectively, which are the sufficient detection limit for practical sensor applications. The obtained results confirm that extended-gate type OFET devices are applicable to enzyme-based biosensors for detecting lactate levels.
\end{abstract}


Keywords: Organic field effect transistor, OFET, lactate, osmium polymer, HRP, lactate oxidase

\section{Introduction}

Over the past two decades, various organic electronic devices such as photovoltaics, transistors and light-emitting diodes have been researched extensively because of their sustainability, lightweight and low consumption of energy (Klauk, 2012). Among these devices, organic field effect transistors (OFETs) have been thus far largely applied to rollable displays (Gelinck et al., 2010). However, interest in OFETs and their advantages have extended beyond electrical engineers and information displays to biomedical engineers and sensor applications. OFETs possess attractive properties such as mechanical flexibility, printability, simple integration and low manufacturing costs; moreover they can be potentially applied to wearable and disposable (or recyclable) biosensors. Accordingly, the research domain for OFET device applications has recently broadened (Lin et al., 2012, Minami et al., 2014 and Torsi et al., 2012), which could allow for novel biosensor device platforms.

Our most recent research efforts have been devoted to the measurement of lactate levels using OFET devices. Lactate is well known as the end product of anaerobic glycolysis, and is being investigated as a biomarker of specific types of cancers (Hirschhaeuser et al., 2011) such as prostate cancer (Tessem et al., 2008), as well as for other critical illnesses (Okorie and Dellinger, 2011 and Zhang et al., 2014). Much of the development work has focused on lactate sensors based on electrochemical and optical 
detection methods (Rassaei et al., 2014 and Wang et al., 2008). Furthermore, an increase of lactate concentration in biological fluids (such as blood and perspiration) is observed during physical exercise, which can be employed as a parameter for monitoring exercise efficiency as well as degrees of wellness (Billat, 1996). While the monitoring of lactate concentration could be carried out via blood sampling, continuous lactate level monitoring is difficult in practice. Hence, the real-time monitoring of lactate levels via perspiration sampling has been investigated as a noninvasive, simple, and potentially dynamic measurement method during exercise (Jia et al., 2013, Khodagholy et al., 2012 and Pribil et al., 2014).

For these reasons, we believe that OFETs are one of the more promising devices for use in lactate sensor applications. Although a non-specific adsorption-based lactate detection using an OFET has been reported (Someya et al., 2002), the development of highly sensitive and selective OFET sensors for the detection of lactate levels is still in their early stages. Here we report on an enzyme-based OFET biosensor that possesses an extended-gate electrode on a flexible plastic film substrate for the purpose of lactate detection and measurement (Fig. 1). Layers of a lactate oxidase and a horseradish peroxidase osmium-redox polymer (Vreeke et al., 1992) were coated onto the electrode, leading to selective, sensitive, and reproducible detection of lactate levels in aqueous media. To the best of our knowledge, this is the first report on lactate sensor device based on extended-gate type OFETs.

\section{Experimental}




\subsection{Reagents and Instruments}

Each of the reagents and solvents used for this study was commercially available and used as supplied. Tetradecylphosphonic acid, L-(+)-lactic acid, the lactate oxidase and glutaraldehyde solution were purchased from Sigma-Aldrich Inc. Cytop ${ }^{\circledR}$ (CTL809M), polyethylene naphthalate (PEN) film, poly(2,5-bis(3-hexadecylthiophene-2yl)thieno[3,2-b]thiophene) (pBTTT-C 16 ), gold, aluminum, FC-43 fluorinert, Teflon ${ }^{\circledR}$ AF1600, 4-(2-hydroxyethyl)-1-piperazineethanesulfonic acid (HEPES) and osmium polymer were purchased from Asahi Glass Co. Ltd., Teijin DuPont Films, Merck KGaA, Tanaka Kikinzoku Kogyo, Furuuchi Chemical Co., 3M Co., Dupont, and Dojindo Laboratories, and Bioanalytical Systems, respectively. p-Cresol, D-(+)-glucose, magnesium chloride, calcium chloride, 1,2-dichlorobenzene, sodium dihydrogenphosphate dihydrate, and disodium hydrogenphoshate were purchased from Wako Pure Chemical Industries. Sodium chloride and urea were purchased from Kanto Chemical Co. Phosphate buffer and HEPES buffer solutions were prepared using Milli$\mathrm{Q}$ water $\left(18 \mathrm{M} \Omega \mathrm{cm}\right.$ at $\left.25^{\circ} \mathrm{C}\right)$.

Metal electrodes were deposited using vacuum evaporation equipment supplied by Cryovac, Co. An oxygen plasma treatment was performed using a PC-300 plasma cleaning system from Samco, Inc. A UV ozone treatment was carried out with a UV253H UV ozone cleaning system from Filgen, Inc. The bank layers were prepared using an IMAGEMASTER 350 dispensing equipment from Musashi Engineering, Inc. The $\mathrm{pH}$ values of solutions were measured using a D-51 $\mathrm{pH}$ meter (Horiba, Ltd.). A silver chloride reference electrode was purchased from BAS, Inc. The electrical 
characteristics for all OFET devices used were measured using a Keithley 2636B source meter.

\subsection{Fabrication of the OFET-based Biosensor Device}

The aluminum ( $\mathrm{Al})$ gate electrode was deposited onto a glass substrate via thermal evaporation (30 $\mathrm{nm}$ thickness). The gate dielectric layer consisted of an aluminumoxide layer $(5 \mathrm{~nm})$ and a tetradecylphosphonic acid $(1.7 \mathrm{~nm})$ self-assembled monolayer (SAM) (Fukuda et al., 2009 and Klauk et al., 2007). The aluminum-oxide layer was formed by treating the $\mathrm{Al}$ gate electrode with the oxygen plasma, with plasma power was $300 \mathrm{~W}$ and the treatment duration was $50 \mathrm{~min}$. The SAM was formed by immersing the substrate in a 2-propanol solution of tetradecylphosphonic acid for $14 \mathrm{~h}$ at room temperature. Au source-drain electrodes $(30 \mathrm{~nm})$ were deposited onto the gate dielectric layer using thermal evaporation and patterned using a shadow-mask. The channel width and length of the OFET device were 1010 and $18 \mu \mathrm{m}$, respectively. To prepare the bank layers, a $1 \mathrm{wt} \%$ solution of an amorphous fluoropolymer in FC-43 was applied using the dispenser equipment. Subsequently, the semiconducting polymer, $\mathrm{pBTTT}^{-\mathrm{C}_{16}}$ (McCulloch et al., 2006 and Umeda et al., 2009) was drop-casted from a 0.03 wt\% solution of 1,2-dichlorobenzene and followed by an anneal at $150{ }^{\circ} \mathrm{C}$ for $30 \mathrm{~min}$ in a nitrogen atmosphere. To passivate the device, Cytop ${ }^{\circledR}$ (CTL-809M) was spin-coated onto the device, followed by a bake at $100{ }^{\circ} \mathrm{C}$ for $10 \mathrm{~min}(100 \mathrm{~nm})$.

The extended-gate electrode consisting of $\mathrm{Au}(50 \mathrm{~nm})$ was prepared on the PEN film substrate (125 $\mu \mathrm{m}$ thick) using thermal evaporation, such that the sensing area for the extended-gate electrode was $15 \mathrm{~mm}^{2}$. By a drop-casting method, the fabricated electrode was coated with a horseradish peroxidase osmium-redox polymer. The coated 
electrode was allowed to stand for $24 \mathrm{~h}$ at $8{ }^{\circ} \mathrm{C}$. After drying out, the electrode was subsequently covered with a mixture of the lactate oxidase from Pediococcus sp. (0.5 units $/ \mu \mathrm{L}$ ) in Dulbecco's phosphate-buffered saline and glutaraldehyde (2 vol\%). To immobilize enzymes on the electrode by the cross-linking agent (=glutaraldehyde), it was allowed to stand for 30 min at room temperature. Finally, the electrode was washed with a HEPES buffer solution (100 mM, pH 7.4).

\subsection{Estimation of the Detection Limit}

In order to determine the limit of detection (LOD) and the limit of quantitation (LOQ) (Miller and Miller, 2010) for lactate, the estimation of these values is derived from intersection of the minimum signal $(=Y)$ and the regression line obtained from the value of $\left(V_{\mathrm{TH}}-V_{\mathrm{TH} 0}\right) / V_{\mathrm{TH} 0}$ in the linear range of the titration curve. The value of $Y$ is estimated by the following equation.

$$
Y=V_{\text {THavg }}-\mathrm{k} \sigma
$$

$V_{\text {THavg }}$ and $\sigma$ are the average value and the standard deviation of the threshold voltage in the absence of lactate, respectively, whereby LOD: $k=3$, LOQ: $k=10$.

\section{Results and Discussion}

\subsection{Electric Characteristics of the Fabricated OFET Device}

To employ OFET devices in biosensor applications, they should be operated at low voltages and made reliable enough for stable and repeated use. Accordingly, the fabricated OFET devices were subjected to repeated measurements of their electrical 
characteristics measured under ambient conditions. The gate voltage $\left(V_{\mathrm{GS}}\right)$ was swept from 0 to $-3 \mathrm{~V}$ while the drain-source voltage $\left(V_{\mathrm{DS}}\right)$ was kept at $-3 \mathrm{~V}$. The field-effect mobility in the saturation region was estimated to be $0.03 \mathrm{~cm}^{2} / \mathrm{Vs}$ and the on/off current ratio was $\sim 100$, values that are sufficient for use in biosensors (Minamiki et al., 2014a, 2014b). More importantly, no hysteresis was observed in the transfer curve and we confirmed that the OFET operated stably under DC bias stress conditions (See the supplementary materials). Based on these results, we confirmed that the fabricated OFET device could operate stably and reliably enough for use in lactate sensor applications.

\subsection{Changes in the OFET Output Current with Increasing Lactate Concentration}

Next, we confirmed that the fabricated OFET sensor device could electrically detect lactate in aqueous media. The gate voltage was applied through a reference electrode $(=\mathrm{Ag} / \mathrm{AgCl})$. Fig. 2 shows time course change of the current $\left(I_{\mathrm{DS}}\right)$ of the OFET biosensor for stepwise changes in the lactate concentration in a HEPES buffer solution at $\mathrm{pH} 7.4$ at room temperature. To remove the initial bias-stress on the device, we operated the OFET $\left(V_{\mathrm{DS}}=V_{\mathrm{GS}}=-2.5 \mathrm{~V}\right)$ for $1 \mathrm{~h}$ before adding lactate. We observed a clear response to the addition of lactate, which suggests that the fabricated OFET sensor is effectively able to monitor the enzymatic reaction. Continuous changes in $I_{\mathrm{DS}}$ were observed by the stepwise addition of lactate, indicating that the OFET sensor could be applied to the real-time monitoring.

\subsection{Changes in the OFET Transfer Characteristics with the Addition of Lactate}


The changes in $I_{\mathrm{DS}}$ show that the field effect modulation of the electrical conductivity in the fabricated OFET device is also affected by the addition of lactate. Fig. 3(a) shows the transfer characteristics of the OFET upon titration with the HEPES solution of lactate. As a result, the lactate titration showed a negative shift of the transfer curve with increasing lactate concentration, while significant changes in the gate current were not observed (see the supplementary materials). This shift is attributed to the change of the hole concentration in the OFET conduction channel. The stability of the gate current suggests that an electrochemical reaction (i.e., electrolysis of water) did not occur by the applied voltage (Fernandes et al., 2010 and Kim et al., 2006). As a result, the enzymatic redox chain reaction on the extended-gate electrode induced the changes in OFET device transfer characteristics (Bergveld, 2003). Fig. 3(b) shows the relationship between the lactate concentration and changes in the threshold voltage $\left(V_{\mathrm{DS}}\right)$. We observed a linear relationship in the region of the low concentration $(0 \sim 1000 \mathrm{nM})$. The LOD and the LOQ were estimated to be $66 \mathrm{nM}$ and $220 \mathrm{nM}$, respectively. The sensitivity of the fabricated OFET sensor device is higher than that of an OFET-based device used for non-selective detection of lactate (Someya et al., 2002) and an ionsensitive inorganic FET-based lactate biosensor (Kharitonov et al., 2001). Furthermore, the estimated sensitivity is comparable to or higher than other electrochemical approaches reported in literature (Ibupoto et al., 2012 and the references therein). The very high level of sensitivity that was obtained allows us to control the sensitivity by shielding the active sites of the lactate oxidase (Pribil et al., 2014) for various applications. We believe that these results will dictate the criteria for the highly sensitive detection of lactate using OFET-based biosensors in the coming future. 


\subsection{Selectivity for Lactate}

When considering the practical application of the developed OFET sensor device to lactate detection in perspiration, high selectivity is also required. The titration of biomolecules or metal ions (which exist in perspiration) with the same concentration of lactate was also carried out. As shown in Fig. 3(c), the addition of $p$-cresol, urea, glucose, sodium chloride, magnesium chloride and calcium chloride resulted in very weak responses (see the details in the supplementary materials). These results strongly indicate that the changes of electrical characteristics in the OFET device are attributed solely to the enzymatic redox reactions on the extended-gate, as we expected.

\section{Conclusion}

In conclusion, we are the first known research group to succeed in demonstrating of an OFET-based biosensor with an enzyme-functionalized extended-gate electrode for sensing lactate levels in aqueous media. The fabricated OFET device could be operated at low voltages and exhibited both high sensitivity and selectivity in the detection of lactate, which are important for use in practical biosensor applications. Moreover, these OFET biosensors can be made ultra-flexible and fabricated with low cost using printing technologies on thin plastic film substrates. Additional research of OFET biosensors for use in lactate detection is being carried in our laboratory.

\section{Acknowledgments}

We gratefully acknowledge the financial support from Japan Science Technology Agency (JST), and Japan Society for the Promotion of Science (JSPS, Grant-in-Aid for Research Activity Start-up, No.26888002). 


\section{Appendix A. Supplementary material}

Supplementary data associated with this article can be found in the online version at $*$.

\section{References}

Bergveld, P., 2003. Sens. Actuators B 88, 1-20.

Bian, C., Tong, J., Sun, J., Zhang, H., Xue, Q., Xia, S., 2011. Biomed. Microdevices 13, $345-352$.

Billat, L.V., 1996. Sports Med. 22, 157-175.

Chen, C.P., Ganguly, A., Lu, C.Y., Chen, T.Y., Kuo, C.C., Chen, R.S., Tu, W.H., Fischer, W.B., Chen, K.H., Chen, L.C., 2011. Anal. Chem. 83, 1938-1943.

Fernandes, E.G.R., Vieira, N.C.S., Queiroz, A.A.A.d., Guimarães, F.E.G., Zucolotto, V., 2010. J. Phys. Chem. C 114, 6478-6483.

Fukuda, K., Hamamoto, T., Yokota, T., Sekitani, T., Zschieschang, U., Klauk, H., Someya, T., 2009. Appl. Phys. Lett. 95, 203301-3.

Gelinck, G., Heremans, P., Nomoto, K., Anthopoulos, T. D., 2010. Adv. Mater. 22, $3778-3798$

Hirschhaeuser, F, Sattler, U.G.A., Mueller-Klieser, W., 2011. Cancer Res. 71, 69216925.

Ibupoto, Z.H., Shah, S.M., Khun, K., Willander, M., 2012. Sensors 12, 2456-2466.

Jia, W.Z., Bandodkar, A.J., Valdes-Ramirez, G., Windmiller, J. R., Yang, Z.J., Ramirez, J., Chan, G., Wang, J., 2013. Anal. Chem. 85, 6553-6560. 
Kharitonov, A.B., Zayats, M., Alfonta, L., Katz, E., Willner, I., 2001, Sens. Actuators B 76, 203-210.

Khodagholy, D., Curto, V.F., Fraser, K.J., Gurfinkel, M., Byrne, R., Diamond, D., Malliaras, G.G., Benito-Lopez, F. Owens, R.M., 2012. J. Mater. Chem. 22, 44404443.

Kim, D.-S., Park, J.-E., Shin, J.-K., Kim, P.K., Lim, G., Shoji, S., 2006. Sens. Actuators B $117,488-494$.

Klauk, H., 2012. Organic Electronics II: More Materials and Applications, Wiley-VCH, Weinheim, Germany.

Klauk, H., Zschieschang, U., Pflaum, J., Halik, M., 2007, Nature 445, 745-748.

Lin, P., Yan, F., 2012. Adv. Mater. 24, 34-51.

Mcculloch, I., Heeney, M., Bailey, C., Genevicius, K., Macdonald, I., Shkunov, M., Sparrowe, D., Tierney, S., Wagner, R., Zhang, W., Chabinyc, M.L., Kline, R.J., Mcgehee, M.D., Toney, M.F., 2006. Nat. Mater. 5, 328-333.

Miller, J.N., Miller, J.C., 2010. Statistics and chemometrics for analytical chemistry, 6th ed. Pearson, Harlow, UK.

Minami, T. Minamiki, T., Hashima, Y., Yokoyama, D., Sekine, T., Fukuda, K., Kumaki, D., Tokito, S., 2014. Chem. Commun. 50, 15613-15615.

Minamiki, T., Minami, T., Kurita, R., Niwa, O., Wakida, S., Fukuda, K., Kumaki, D., Tokito, S., 2014a. Appl. Phys. Lett. 104, 243703.

Minamiki, T., Minami, T., Kurita, R., Niwa, O., Wakida, S., Fukuda, K., Kumaki, D., Tokito, S., 2014b. Materials 7, 6843-6852.

Okorie, O.N., Dellinger, P., 2011. Crit. Care Clin. 27, 299-326. 
Pribil, M.M., Laptev, G.U., Karyakina, E.E., Karyakin, A.A., 2014. Anal. Chem. 86, $5215-5219$.

Rassaei, L., Olthuis, W., Tsujimura, S., Sudhölter, E.J.R., van den Berg, A., 2014. Anal. Bioanal. Chem. 406, 123-137.

Someya, T., Dodabalapur, A., Gelperin, A., Katz, H.E., Bao, Z., 2002. Langmuir 18, $5299-5302$.

Tessem, M.-B.; Swanson, M. G.; Keshari, K. R.; Albers, M. J.; Joun, D.; Tabatabai, Z. L.; Simko, J. P., Shinohara, K., Nelson, S. J., Vigneron, D. B., Gribbestad, I. S., Kurhanewicz, J., 2008. Magn. Reson. Med. 60, 510-516.

Torsi, L., Magliulo, M., Manoli, K., Palazzo, G., 2013. Chem. Soc. Rev. 42, 86128628.

Umeda, T., Kumaki, D., Tokito, S., 2009. J. Appl. Phys. 105, 024516-1-5.

Vreeke, M., Maidan, R., Heller, A., 1992. Anal. Chem. 64, 3004-3090.

Wang, Y., Xu, H., Zhang, J.M., Li, G., 2008. Sensors 8, 2043-2081.

Zhang, Z., Xu, X., Chen, K. 2014. BMJ Open 4, e004752. 


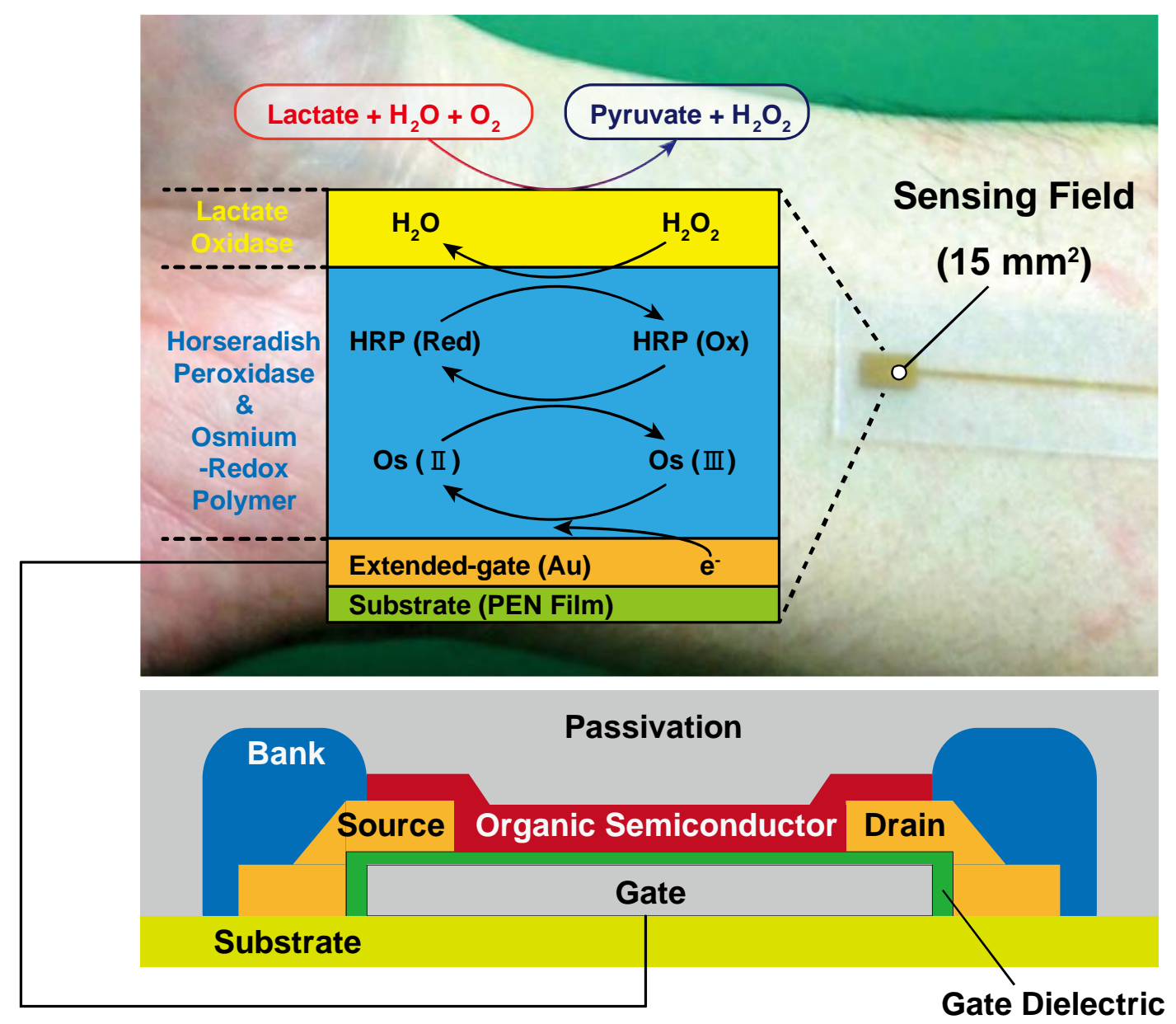

Fig. 1. Schematic illustration of the extended-gate type OFET biosensor for detecting lactate and a photograph of the fabricated extended-gate. 


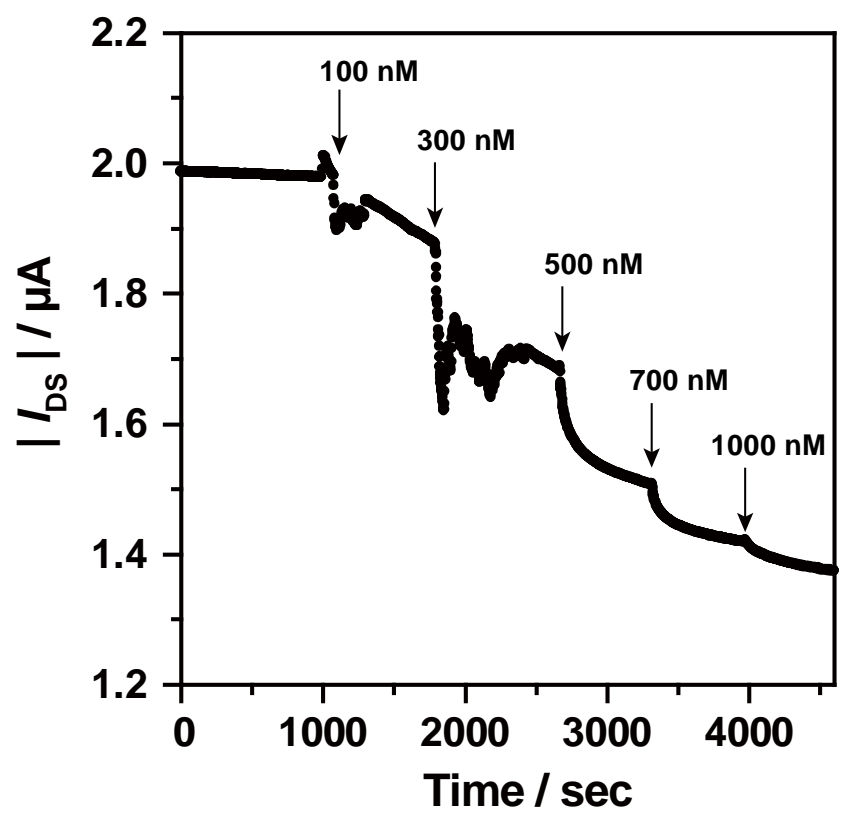

Fig. 2. Time course change of the drain current $\left(I_{\mathrm{DS}}\right)$ with increasing concentration of lactate. $V_{\mathrm{DS}}=V_{\mathrm{GS}}=-2.5 \mathrm{~V}$. 
a)

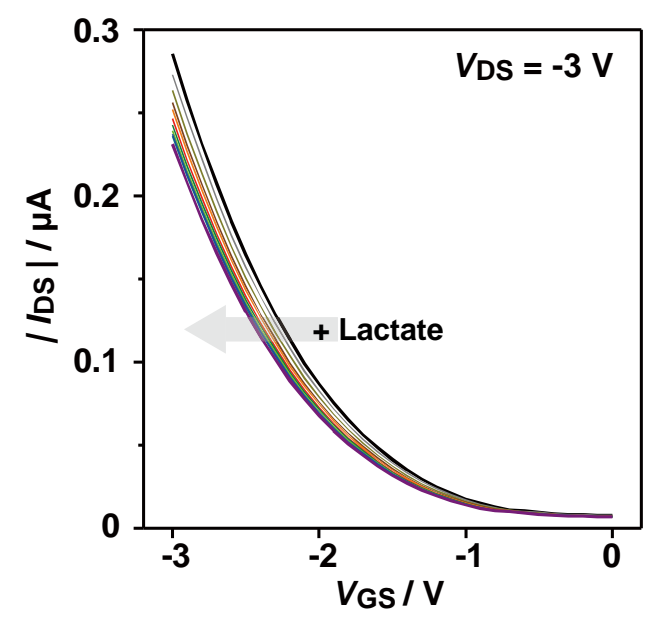

C) b)

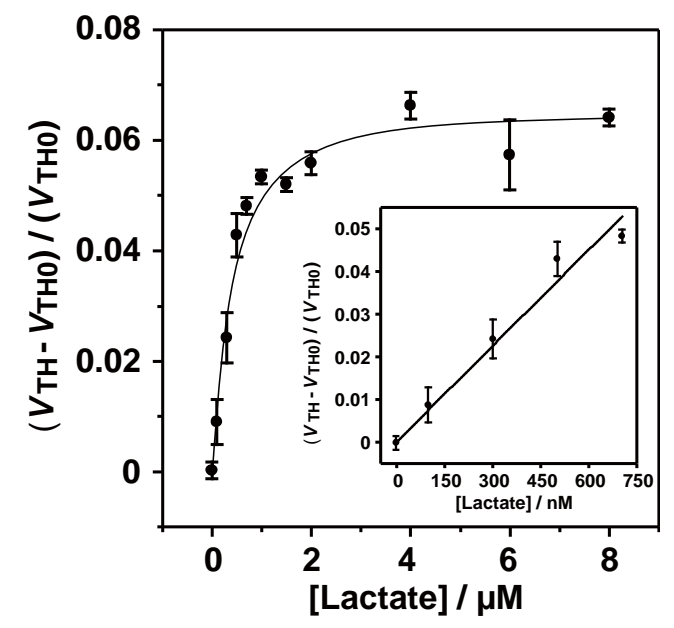

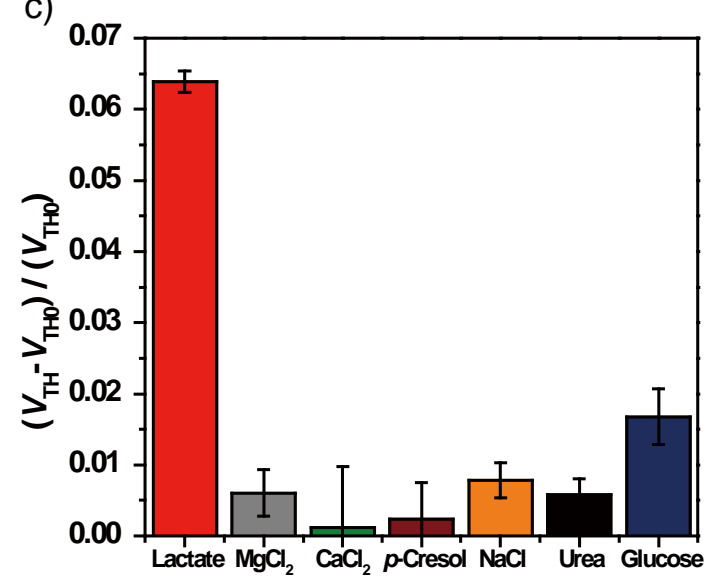

Fig. 3. (a) Transfer characteristics $\left(I_{\mathrm{DS}}-V_{\mathrm{GS}}\right)$ of the OFET sensor upon titration with lactate in a HEPES-buffer solution $(100 \mathrm{mM})$ at $\mathrm{pH} 7.4$ at room temperature. $V_{\mathrm{DS}}$ $=-3 \mathrm{~V}$. [Lactate] $=0-8 \mu \mathrm{M}$. (b) Changes in threshold voltage $\left(V_{\mathrm{TH}}\right)$ of the OFET device by adding lactate at various concentrations in a HEPES-buffer solution $(100 \mathrm{mM})$ at $\mathrm{pH} 7.4$ at room temperature. The inset shows the lower end of the titration. (c) Changes in the threshold voltage $\left(V_{\mathrm{TH}}\right)$ of the OFET device by the addition of various analytes $(8 \mu \mathrm{M})$ in a HEPES-buffer solution $(100 \mathrm{mM})$ at $\mathrm{pH}$ 7.4 at room temperature. 
TOC

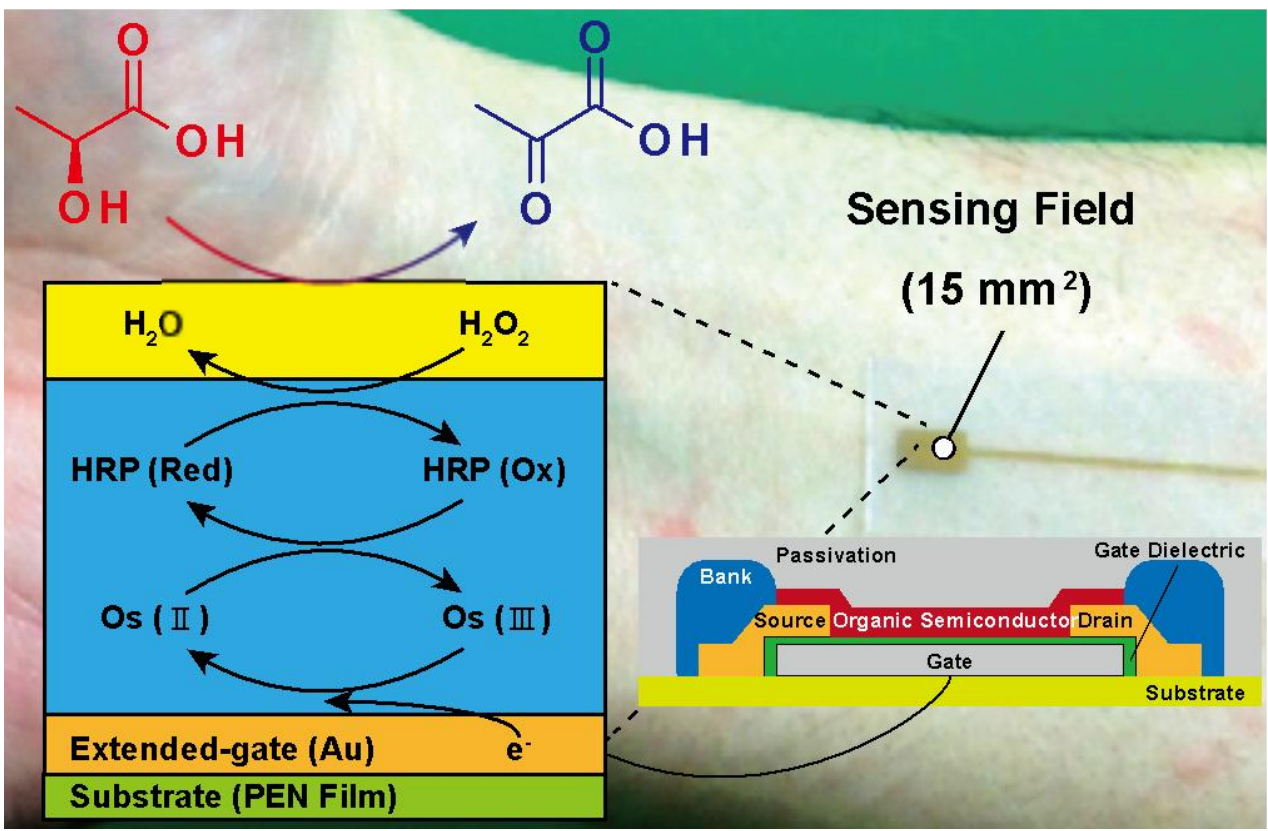

